\title{
Preparation, Characterization, and Properties of Polyurethane-Grafted Multiwalled Carbon Nanotubes and Derived Polyurethane Nanocomposites
}

\author{
Tzong-Liu Wang, ${ }^{1}$ Chin-Chung Yu, ${ }^{2}$ Chien-Hsin Yang, ${ }^{1}$ Yeong-Tarng Shieh, ${ }^{1}$ \\ Yu-Zen Tsai, ${ }^{3}$ and Na-Fu Wang ${ }^{3}$ \\ ${ }^{1}$ Department of Chemical and Materials Engineering, National University of Kaohsiung, Kaohsiung 811, Taiwan \\ ${ }^{2}$ Department of Applied Physics, National University of Kaohsiung, Kaohsiung 811, Taiwan \\ ${ }^{3}$ Department of Electronics, Cheng Shiu University, Kaohsiung County 833, Taiwan
}

Correspondence should be addressed to Tzong-Liu Wang, tlwang@nuk.edu.tw

Received 15 July 2011; Accepted 3 October 2011

Academic Editor: Gaurav Mago

Copyright (C) 2011 Tzong-Liu Wang et al. This is an open access article distributed under the Creative Commons Attribution License, which permits unrestricted use, distribution, and reproduction in any medium, provided the original work is properly cited.

\begin{abstract}
We incorporated hydroxyl groups into the polyurethane backbone and then used the "grafting to" approach to functionalize the multiwalled carbon nanotubes (MWNTs) via the esterification reaction between MWNTs and segmented polyurethanes (PUs). X-ray photoelectron spectroscopy (XPS) spectra showed that the sidewalls of MWNTs had been functionalized with acid treatment, and the amount of $\mathrm{COOH}$ increased with increasing acid treatment time. FTIR spectra further confirmed that PU was covalently attached to the sidewalls of MWNTs. The functionalized acid amount and the grafted PU amount were determined by thermogravimetric analyses (TGAs). Comparative studies based on SEM images of the PU-functionalized and chemically defunctionalized MWNT samples also revealed the covalent coating character. Dynamic mechanical analysis (DMA) of nanocomposite films prepared from PU and PU-functionalized MWNTs showed enhanced mechanical properties and increased soft segment $T_{g}$. Tensile properties indicated that PU-functionalized MWNTs were effective reinforcing fillers for the polyurethane matrix.
\end{abstract}

\section{Introduction}

In recent decades, polymer-carbon nanotube composite materials have attracted much attention for their potential applications in unique lightweight materials with distinctly superior mechanical, thermal, and electronic properties [1-4]. This can be attributed to the fascinating electronic, thermal, and mechanical properties of carbon nanotubes (CNTs) [5, 6]. With extremely high mechanical strength and chemical stability, CNTs represent attractive possibilities for developing ultrastrong composite materials [7, 8]. The effective utilization of CNTs in nanocomposite applications depends strongly on the ability to disperse the CNTs homogeneously throughout a matrix without destroying the integrity of the CNTs. The CNTs can offer a kind of nanosize reinforcement with a light weight, a hollow-core immerse aspect ratio, and an exceptionally high axial strength. Hence, significant efforts have been made in the fabrication of these nanocomposites by dispersing either single-walled (SWNT) or multiple-walled (MWNT) carbon nanotubes into various polymer matrices.

However, the manipulation and processing of CNTs have been limited by their insolubility in most common solvents [9]. By functionalization or modification of nanosurfaces of CNTs, it has unlocked a new era in the development and applications of CNTs containing hybrid nanomaterials [1012]. This can be generally fulfilled by the "grafting to" [13$16]$ and "grafting from" [17-20] approaches. Some experimental studies on CNT-reinforced polymer composites have been reported for various kinds of organic polymers, including polyethylene $[21,22]$ polypropylene [23], poly(methyl methacrylate) $[24,25]$, polystyrene [26], pitch [27], and epoxy $[28,29]$, with enhanced mechanical and electrical properties. More recently, polyurea-functionalized CNTs have 

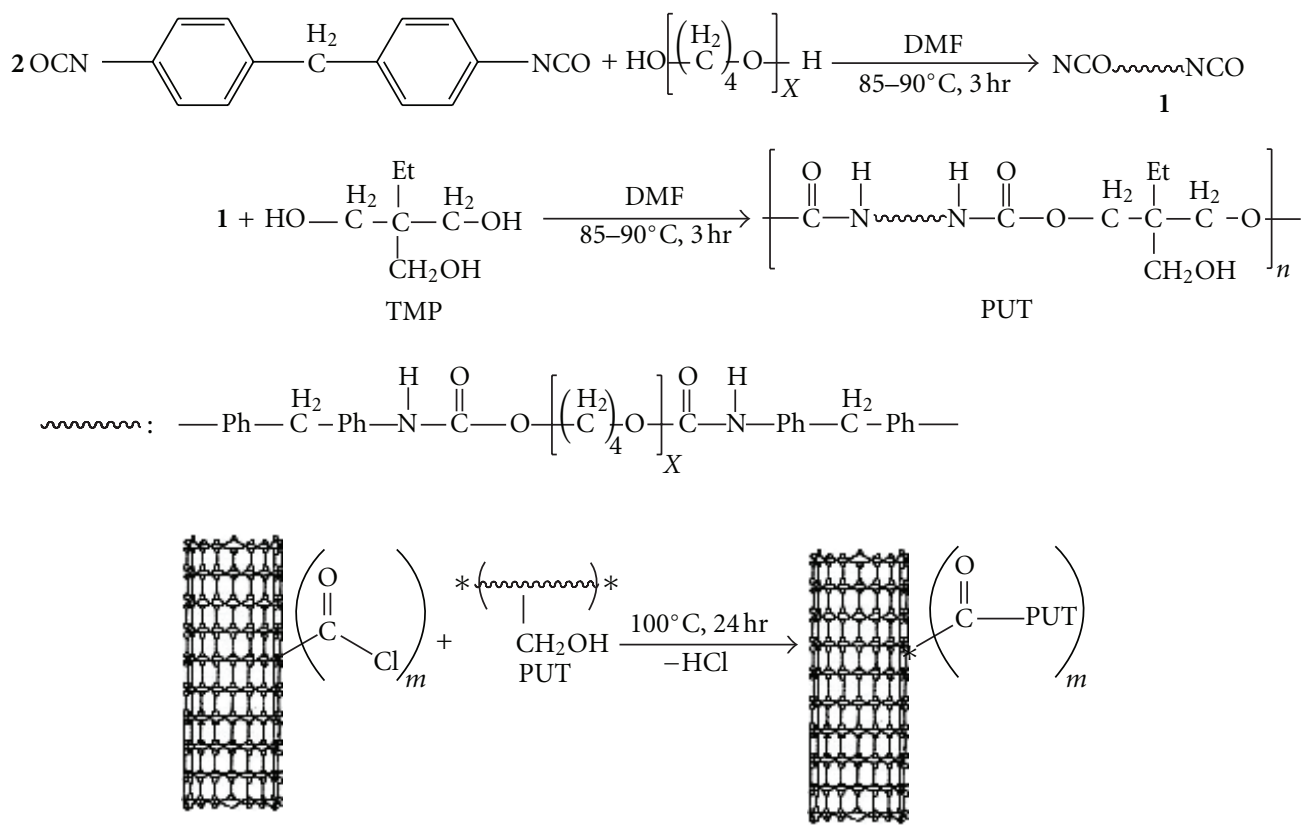

Scheme 1: Synthetic route for the functionalization of MWNTs with segmented polyurethanes (PUT).

also been prepared via an in situ polycondensation approach [30]. In a similar manner, polyurethane-functionalized SWNTs have also been prepared through a two-step reaction [31]. However, Gao's and Xia's approaches could not be used in the preparation of segmented polyurethane elastomers functionalized carbon nanotubes, because polyurethane elastomers must be prepared through a prepolymer technique. In addition, although Kwon and Kim reported dispersion of CNTs in a waterborne polyurethane matrix $[32,33]$, the dispersion of carbon nanotubes in the polyurethane elastomer matrix was only through a noncovalent solution blending.

Elastomeric thermoplastic block copolymers are typically microphase-separation materials containing two types of segments in their molecular architecture. It was expected CNTs functionalized with polyurethane elastomers (i.e., segmented copolyurethanes or polyurethane block copolymers) via a covalent bonding would be more compatible with polyurethane elastomer matrices and hence could reinforce polyurethane elastomers. Since polyurethane block copolymers are a class of high-performance materials for versatile end use, nanocomposites prepared from polyurethane elastomers reinforced with CNTs may extend its application in various fields.

In a previous report, we have presented a methodology to bind as-prepared segmented polyurethanes to CNTs via the "grafting to" approach [34]. In this work, we incorporated hydroxyl groups into the polyurethane backbone and then used the "grafting to" approach to functionalize the MWNTs via the esterification reaction between MWNTs and PU. The segmented polyurethanes with hydroxyl groups pendant on the chain extender were synthesized by the conventional prepolymer technique. The functionalized CNTs and MWNTPU nanohybrids have been characterized to confirm the covalent linkage. In addition, results from the fabrication

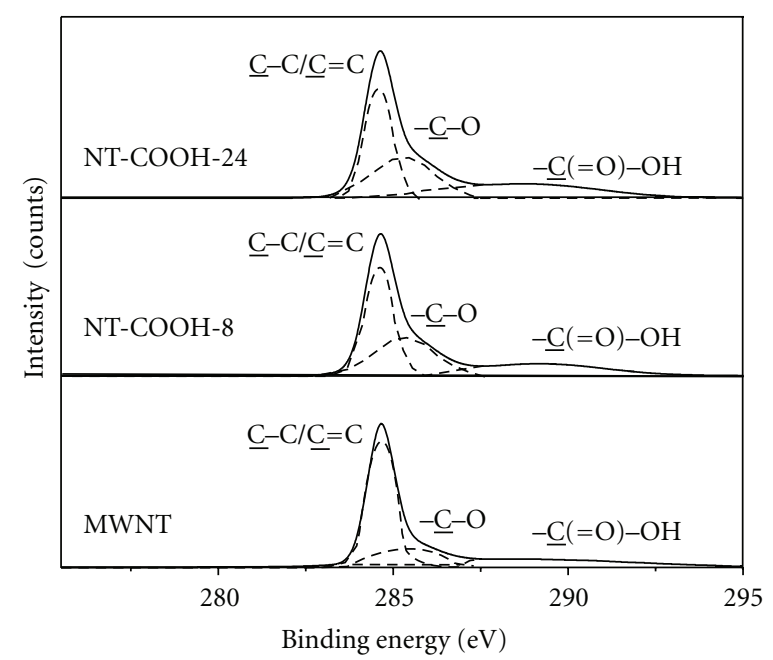

FIGURE 1: High-resolution XPS spectra of crude MWNTs and acidtreated MWNTs with different treatment times.

and characterization of the polyurethane-carbon nanotube nanocomposite films are presented and discussed.

\section{Experimental}

2.1. Materials. The MWNTs used in this work were purchased from Desunnano Co., Ltd.; the purity is higher than 95\%. Thionyl chloride $\left(\mathrm{SOCl}_{2}\right)$ was obtained from Aldrich and used as received. 4,4' -Methylenebis(phenyl isocyanate) (MDI, Aldrich), methyl isobutyl ketone (MIBK, Hayashi Chemicals), N,N-dimethylformamide (DMF, Tokyo Chemicals), ethyl acetate (EA, Tokyo Chemicals), and dimethyl sulfoxide (DMSO, Nacalai Tesque, Inc.) were distilled under 
reduced pressure. 1,1,1-Tris(hydroxymethyl)propane (TMP, Fluka) and tetrahydrofuran (THF, Tokyo Chemicals) were used as received. Poly(tetramethylene ether glycol) (PTMG, $\overline{\mathrm{Mn}}=1000$ ) was degassed in vacuo at $55^{\circ} \mathrm{C}$ and $600 \mathrm{~Pa}$ $(4.5 \mathrm{mmHg})$ for $3 \mathrm{~h}$ to remove any absorbed water. Nitric acid (EP grade), and sulfuric acid (EP grade) were purchased from Nihon Shiyaku Industries, Ltd. and used as received.

\subsection{Synthesis of the Segmented Polyurethane Elastomer (PUT).}

The preparation method of the segmented polyurethane elastomer carrying a pendant hydroxyl group in the chain extender has been described in our previous article [34]. GPC (DMF): $\overline{\mathrm{Mn}}=31,300 \mathrm{~g} / \mathrm{mol}, \overline{\mathrm{Mw}}=51,000 \mathrm{~g} / \mathrm{mol}$, and PDI $=1.63$. The reaction is given in Scheme 1. For convenience, this polymer is designated as PUT.

2.3. Acid Treatment and Acylation of MWNTs. The detail procedures have been published previously [34]. The samples (MWNT-COOH) obtained for acid treatment of 8 and $24 \mathrm{~h}$ were designated as NT-COOH- 8 and NT-COOH-24, respectively. After acylation, the samples (MWNT-COCl) were washed with purified EA three times and ready for esterification with PUT.

2.4. Esterification of Acylated MWNTs with PUT. $0.2 \mathrm{~g}$ of as-prepared MWNT-COCl was immediately reacted with $1.0 \mathrm{~g}$ of PUT at $100^{\circ} \mathrm{C}$ for $24 \mathrm{~h}$, obtaining PUT-grafted MWNTs after repeated centrifugation at $7500 \mathrm{rpm}$, washing, and vacuum drying. The products (MWNT-PUT) prepared from acylation of NT-COOH-8 and NT-COOH-24 were designated as NT-PUT-8 and NT-PUT-24, respectively.

2.5. Preparation of Nanocomposite Films. In a typical experiment, the matrix PUT $(4 \mathrm{~g})$ was dissolved in DMF $(16 \mathrm{~mL})$ to form a homogeneous solution. To the solution was added dropwise a DMF solution of PUT-functionalized carbon nanotubes (NT-PUT-8) under constant stirring. The resulting solution was cast onto a glass substrate and dried at $50^{\circ} \mathrm{C}$ for $48 \mathrm{~h}$. In this study, three different compositions, that is, $1 \mathrm{wt} \%, 5 \mathrm{wt} \%$, and $10 \mathrm{wt} \%$ of NT-PUT- 8 based on the original amount of PUT ( $4 \mathrm{~g})$ were prepared for comparison. The composite films were designated as PUT/NT-PUT-8-1, PUT/NT-PUT-8-5, and PUT/NT-PUT-8-10, respectively.

2.6. Characterization. Infrared spectra of samples were obtained using a Bio-Rad FTS 165 Fourier transform infrared spectrometer. The spectra were obtained over the frequency range of 4000 to $400 \mathrm{~cm}^{-1}$ at a resolution of $4 \mathrm{~cm}^{-1}$.

XPS surface analysis was carried out using a VG Instruments X-ray photoelectron spectrometer. $\mathrm{Mg}-\mathrm{K} \alpha$ radiation was used as the X-ray source and the photoelectron peaks (in the wide-scan spectra) from the samples were numerically fitted using Lorentzian curves with an integral background subtraction and analyzed at an angle of $45^{\circ}$ to the surface. The adventitious $\mathrm{C}(1 \mathrm{~s})$ signal at $284.6 \mathrm{eV}$ was used to calibrate the charge-shifted energy scale.

Thermogravimetric analysis (TGA) experiments were carried out on samples placed in a platinum sample pan

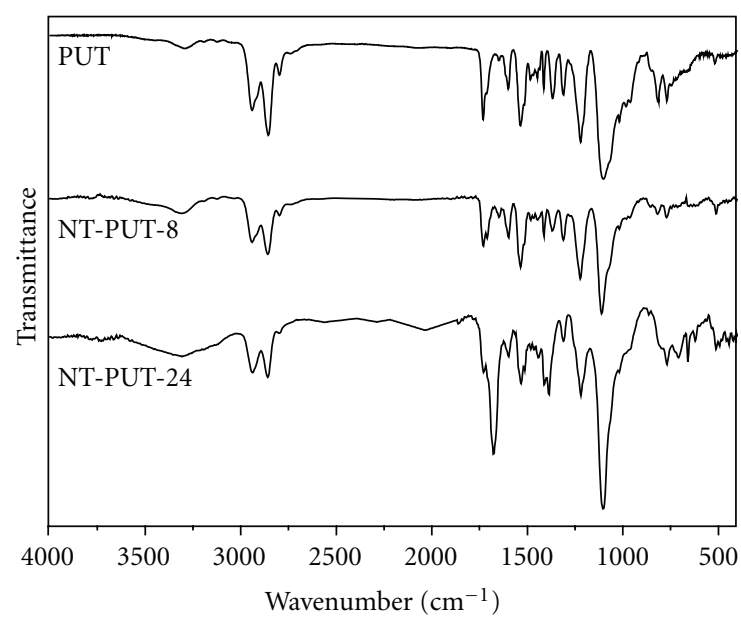

FIGURE 2: FTIR spectra of PUT, NT-PUT-8, and NT-PUT-24.

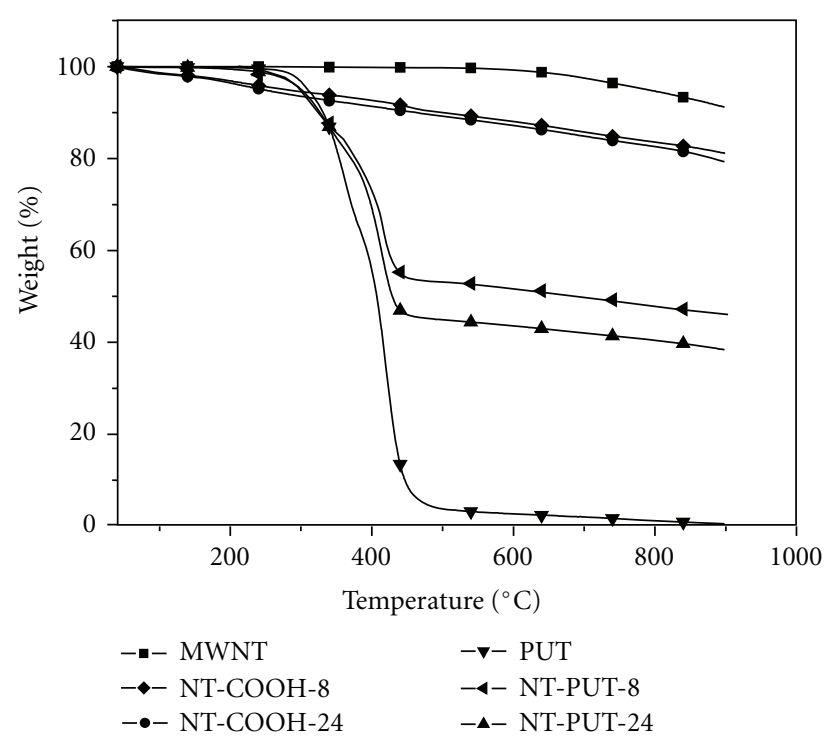

FIGURE 3: TGA weight loss curves of crude MWNTs, acid-treated MWNTs, and PUT-functionalized MWNTs under a nitrogen atmosphere.

using a TA Instruments SDT-2960 analyzer. Products ranging from 4 to $5 \mathrm{mg}$ were loaded into the platinum pan and sealed in the sample chamber. The samples were heated from $50^{\circ} \mathrm{C}$ to $900^{\circ} \mathrm{C}$ under a nitrogen atmosphere at a heating rate of $10^{\circ} \mathrm{C} / \mathrm{min}$.

Scanning electron microscopy (SEM) images were recorded using a Hitachi S-4800 field-emission microscope, and the samples were precoated with a homogeneous gold layer by sputtering technology.

Dynamic mechanical analysis (DMA) was performed on a Perkin Elmer DMA7e unit with an operating temperature range of $-100 \sim 50^{\circ} \mathrm{C}$. The heating rate was set at $5^{\circ} \mathrm{C} / \mathrm{min}$. The sample size was approximately $5.5 \times 1.5 \times 0.5 \mathrm{~mm}^{3}$.

Stress-strain data of nanocomposite films were obtained using a Universal Testing Machine (Shimadzu AGS-500A Series) with a $10 \mathrm{~kg}$ load cell and film grips. The crosshead 

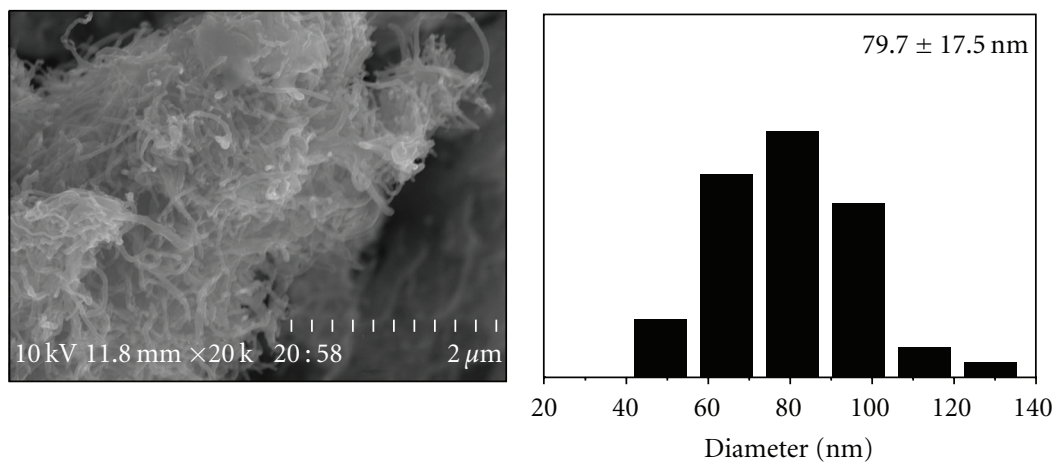

(a)
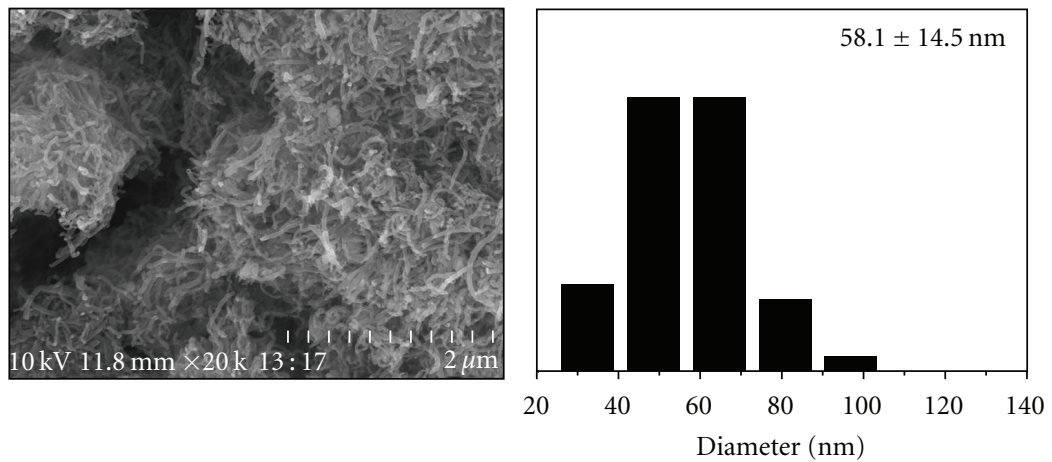

(b)
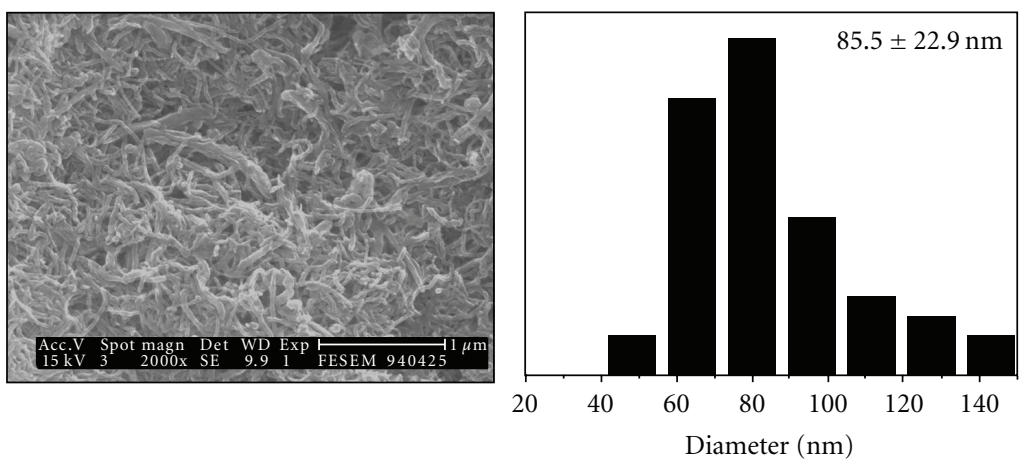

(c)
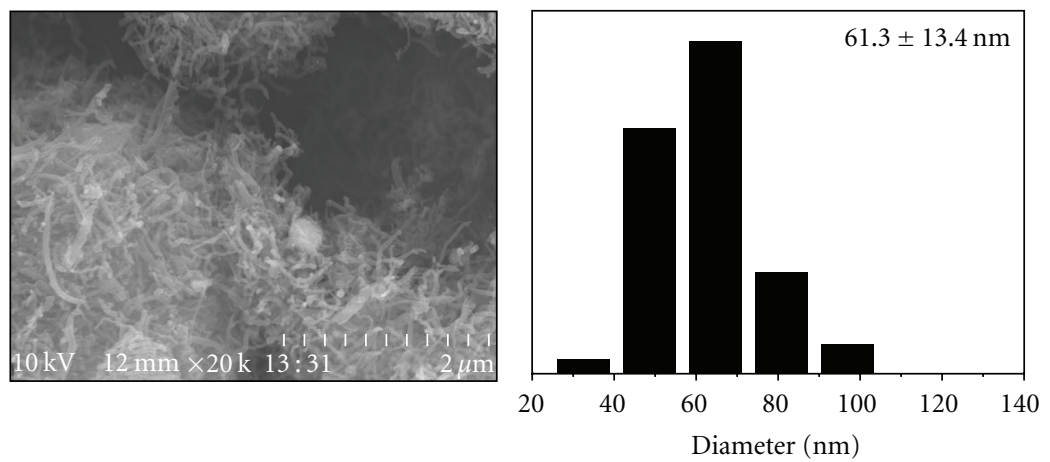

(d)

FigURE 4: SEM micrographs of PUT-functionalized and chemically defunctionalized MWNT samples. (a) NT-PUT-8. (b) NT-PUT-8 defunctionalized at $900^{\circ} \mathrm{C}$ for $2 \mathrm{~h}$. (c) NT-PUT-24. (d) NT-PUT-24 defunctionalized at $900^{\circ} \mathrm{C}$ for $2 \mathrm{~h}$. 


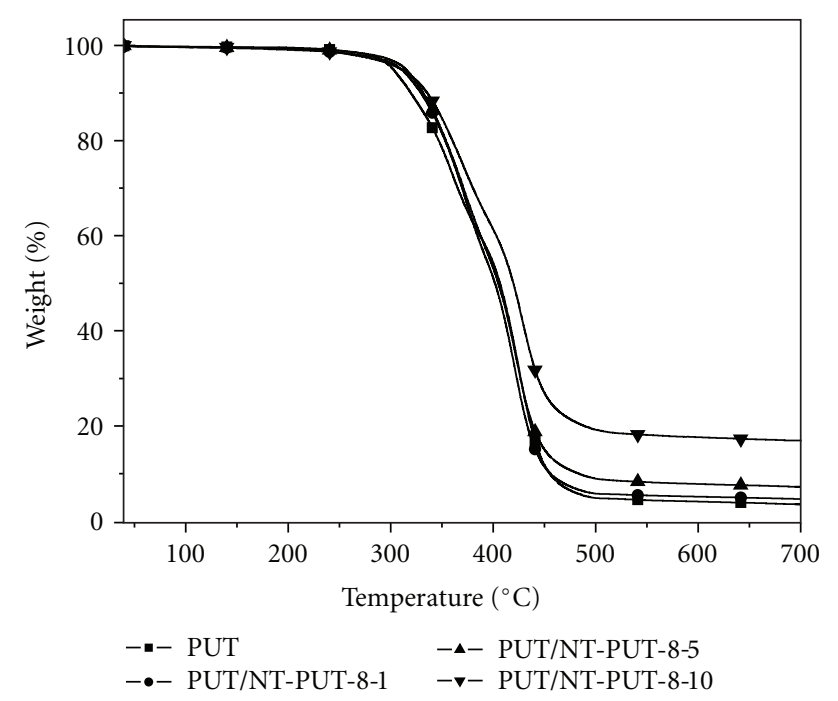

FIgUre 5: TGA weight loss curves of PUT and PUT/NT-PUT-8 nanocomposites under a nitrogen atmosphere.

speed was $20 \mathrm{~mm} / \mathrm{min}$. Measurements were made at room temperature using a $1.2 \times 0.4 \mathrm{~cm}^{2}$ dumbbell sample.

\section{Results and Discussion}

3.1. X-Ray Photoelectron Spectroscopy (XPS) Analysis of AcidTreated MWNTs. In our previous article, Raman spectroscopy has showed that the suitable time of acid treatment was ca. $8 \mathrm{~h}$ [34]. Therefore, our polymeric carbon nanocomposites discussed here and after were all prepared from NT-PUT8 in view of processing and application.

In the present study, electron spectroscopy for chemical analysis (i.e., XPS) was further used to provide qualitative and quantitative information about the elemental composition of acid-treated MWNTs $[35,36]$. In the high-resolution spectra of $\mathrm{C}(1 \mathrm{~s})$, as shown in Figure 1, it is evident that the $\mathrm{C}(1 \mathrm{~s})$ core level spectra of acid-treated MWNTs consist of three well-resolved peaks after curve-fitting. The major peak, referenced to $284.6 \mathrm{eV}$ ( $\mathrm{C}-\mathrm{C}$ or $\mathrm{C}=\mathrm{C})$, is ascribed as unsubstituted aromatic carbon in carbon nanotubes. The peak shifted approximately $0.7 \mathrm{eV}$ (i.e., at $285.3 \mathrm{eV}$ ) toward the higher binding energy side of the main peak corresponds to the carbon singly bound to oxygen $(\mathrm{C}-\mathrm{O})$. This is probably due to the residual oxygen after the purification step of pristine MWNTs or ether-type oxygen (-C-O-) of $\mathrm{COOH}$. The small peak, present at $288.8 \mathrm{eV}$, can be attributed to the carbon double-bonded $(\mathrm{C}=\mathrm{O})$ to oxygen in the carboxylic acid groups. The quantitative results of XPS are listed in Table 1 . The higher surface oxygen content for the carboxylic acid functionalized MWNTs, NT-COOH-8, and NT-COOH24 , in comparison with that of the pristine materials, is evidence of $\mathrm{COOH}$ groups on the MWNT surface.

3.2. Synthesis and Characterization of MWNT-Polyurethane Nanohybrids. Through the "grafting to" approach, the MWNTs were functionalized by grafting polyurethane to the
TABLE 1: (1) XPS peak position (in eV) and (2) percentage (in atom$\%)$ from high-resolution C(1s) spectra of crude MWNTs and acidtreated MWNTs.

\begin{tabular}{lcccc}
\hline Sample & & $\underline{\mathrm{C}}-\mathrm{C} / \underline{\mathrm{C}}=\mathrm{C}$ & $\underline{\mathrm{C}}-\mathrm{O}$ & $\underline{\mathrm{C}}(=\mathrm{O})-\mathrm{OH}$ \\
\hline \multirow{2}{*}{ MWNT } & $(1)$ & $284.6 \mathrm{eV}$ & $285.4 \mathrm{eV}$ & $288.6 \mathrm{eV}$ \\
& $(2)$ & $76.65 \%$ & $16.55 \%$ & $6.80 \%$ \\
\hline \multirow{2}{*}{ NT- } & $(1)$ & $284.6 \mathrm{eV}$ & $285.3 \mathrm{eV}$ & $289.1 \mathrm{eV}$ \\
COOH-8 & $(2)$ & $42.57 \%$ & $33.44 \%$ & $23.99 \%$ \\
\hline \multirow{2}{*}{ NT- } & $(1)$ & $284.6 \mathrm{eV}$ & $285.3 \mathrm{eV}$ & $288.7 \mathrm{eV}$ \\
COOH-24 & $(2)$ & $39.74 \%$ & $34.05 \%$ & $26.21 \%$ \\
\hline
\end{tabular}

sidewalls of MWNTs (Scheme 1). The chemical structure of the resulting MWNT-polyurethane nanohybrids is also illustrated in Scheme 1. It is noteworthy that the adsorbed polyurethane can be efficiently removed from the products by filtration and washing as mentioned in Section 2. From IR measurements for the upper layer of DMF solution, collected by centrifuging ( $1 \mathrm{~h}$ at a rate of $7500 \mathrm{rpm}$ ) of MWNT-PUT samples from the solution it is shown that no polyurethane signals appeared in the spectrum. This indicates that the adsorbed polymer quantity is negligible. Therefore, the "grafting to" approach presented here promised the grafting of polyurethanes onto CNT surfaces with some extent of control.

The molecular composition of the resulting MWNT-polyurethane nanohybrids was confirmed by FTIR measurements. The IR spectra of NT-PUT-8 and NT-PUT-24 are shown in Figure 2. For both samples, the characteristic absorption peaks of polyurethane such as $-\mathrm{CH}_{2}-$, NHCOO-, and $\mathrm{C}-\mathrm{O}-\mathrm{C}$, and clearly appear at 2930/2853, 1730, $1100 \mathrm{~cm}^{-1}$, respectively. The benzene-ring $\mathrm{C}=\mathrm{C}$ absorption peak from MDI is at ca. $1600 \mathrm{~cm}^{-1}$, while the peaks at $1530 \mathrm{~cm}^{-1}$ and $1220 \mathrm{~cm}^{-1}$ correspond to $\mathrm{C}-\mathrm{N}$ of urethane groups. This spectrum clearly shows that PUT has been grafted to MWNTs successfully.

In general, polymer-functionalized CNTs would show much higher solubility or better dispersibility as compared with pristine nanotubes. Herein, as polyurethane is polar, our resulting samples of MWNT-polyurethane are readily dispersed in polar organic solvents such as DMSO, DMF, 1-methyl-2-pyrrolidinone (NMP), and N,N-dimethyl acetamide (DMAc). The clear, gray solutions from the soluble samples are stable, without any precipitations over time.

\subsection{Thermal Analysis of the Resulting MWNT-Polyurethane} Nanohybrids. In order to obtain the grafted amount of polyurethane on MWNTs, thermal analyses of MWNT-PUT prepared from MWNTs with different times of acid treatment $(8 \mathrm{~h}$ and $24 \mathrm{~h})$ were carried out. The thermal stability for crude MWNTs and functionalized MWNTs in nitrogen is illustrated in Figure 3. As seen in the figure, the TGA curves display a two-step degradation mechanism for PUT, NT-PUT-8, and NT-PUT-24, which is quite different with the one-step degradation mechanism of MWNT and acidtreated MWNTs (MWNT-COOH, i.e., NT-COOH-8 and NT-COOH-24). It is obvious that PUT has been grafted 


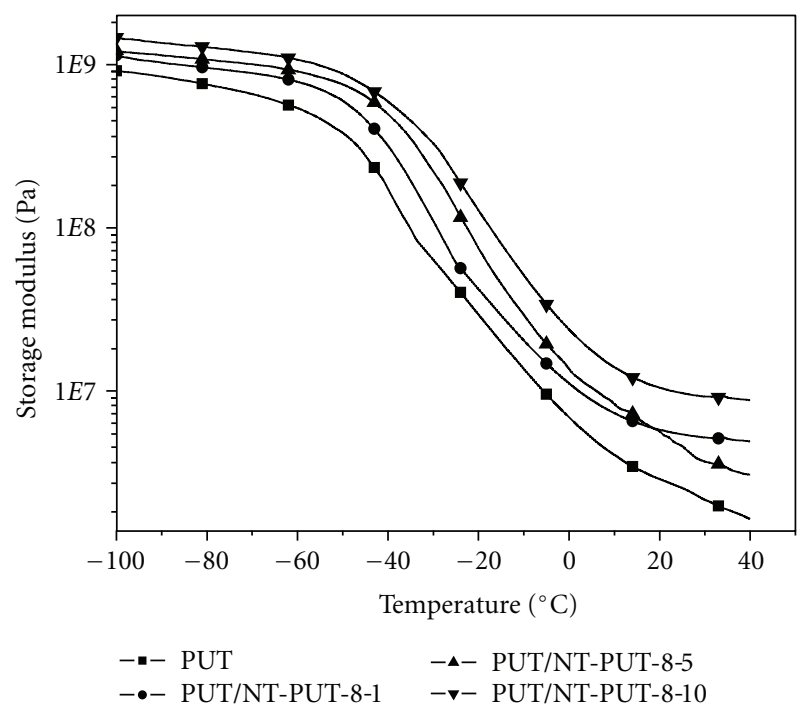

(a)

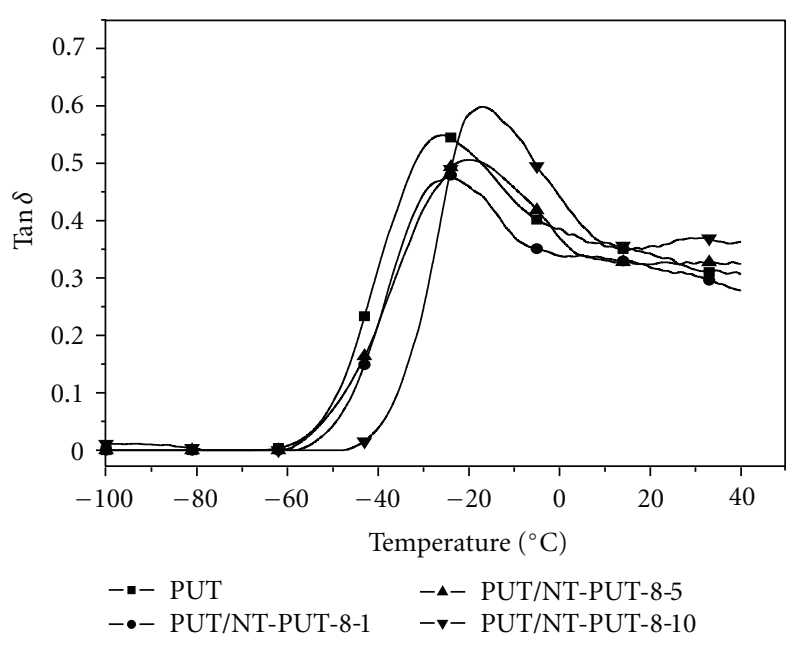

(b)

FIGURE 6: Temperature dependence of (a) storage modulus ( $\left.E^{\prime}\right)$; (b) loss tangent ( $\tan \delta$ ) for PUT and PUT/NT-PUT-8 composite films.

onto the sidewalls of MWNTs. In the MWNT-COOH case, there is a continuous but not very obvious decrease in weight, which is typical for acid functionalized MWNTs. In comparison with the curves of MWNTs and acid-treated MWNTs, the rapid degradation stage in NT-PUT-8 and NT-PUT-24 may arise due to the decomposition of grafted PUT. As compared to crude MWNTs, the functionalized acid amount could be calculated by the subtraction of char yields of acid-treated MWNTs from pristine MWNT. Thus, the functionalized acid amounts for NT-COOH-8 and NTCOOH-24 are 10.0 and $12.2 \%$, respectively. It is evident that the increased acid amount after acid treatment for $8 \mathrm{~h}$ is not much, demonstrating that longer acid-treated time may be not necessary. Therefore, to save the processing time for the application, our nanocomposites were only prepared from NT-PUT-8 nanohybrid. In addition, the grafted amounts of PUT were also obtained. According to the TGA traces, the PUT contents in the NT-PUT- 8 and NT-PUT-24 samples are approximately $35.6 \%$ and $42.2 \%$, respectively. It was found that the longer acid treatment time results in a higher grafted amount of PUT. This further confirms the successful functionalization of MWNTs.

3.4. Morphology of the MWNT-Polyurethane Nanohybrids. The fine nanostructures of the as-prepared MWNT-polyurethane nanohybrides were investigated by SEM. As shown in Figures 4(a) and 4(c), MWNTs were coated by a layer of polymer chains. From the SEM images of the PUTgrafted MWNT samples, we can clearly discern that the higher the quantity of the grafted polymer, the thicker the polymer shells. The calculated diameters for NT-PUT-8 and NT-PUT-24 are ca. 79.7 and $85.5 \mathrm{~nm}$, respectively. After heat treatment of NT-PUT- 8 and NT-PUT- 24 at $900^{\circ} \mathrm{C}$ for $2 \mathrm{~h}$, the defunctionalized tube surfaces are relatively smooth and clean (Figures 4(b) and 4(d)), obviously different from those of the polyurethane-functionalized MWNTs. The tube diameters are ca. 58.1 and $61.3 \mathrm{~nm}$, respectively, being close to the diameter of pristine MWNTs.

3.5. Preparation and Thermal Analysis of Polyurethane Nanocomposites. In this research, MWNTs were functionlalized with PUT in order to be more compatible with the polymer matrix, PUT, of the nanocomposites. Due to the urethane groups of PUT, there are strong intermolecular interactions arising from the hydrogen bondings between PUT and MWNT-PUT nanohybrids, resulting in high compatibility between both components. The common solubility of the PUT-functionalized carbon nanotubes and the matrix PUT also makes the solution casting easier. The compatibility of the PUT-functionalized carbon nanotubes with polyurethane and the dispersion of the nanotubes in polyurethane matrix were evaluated via the fabrication of nanocomposite thin films. The PUT-functionalized carbon nanotubes used in this study are NT-PUT- 8 as mentioned previously. In a typical experiment, a calculated amount of NT-PUT-8 (w/w) based on PUT matrix was dissolved in DMF and added to a PUT solution. For comparison, 1, 5, and $10 \mathrm{wt} \%$ of NT-PUT- 8 based on $100 \mathrm{wt} \%$ of PUT were used as the reinforcing fillers. The nanocomposites prepared from PUT reinforced with the aforementioned amounts of NT-PUT- 8 were designated as PUT/NT-PUT-8-1, PUT/NTPUT-8-5, and PUT/NT-PUT-8-10, respectively. The resulting composite solution was allowed to settle overnight and then centrifuged to remove any residual insoluble species, followed by being concentrated to attain the desired viscosity. The viscous but transparent solution was used for the casting of a thin film. The polyurethane-MWNT composite thin film thus obtained is transparent with a high optical quality. The successful fabrication of optically high-quality nanocomposite thin films reflects the excellent compatibility of the PUT-functionalized carbon nanotubes with polyurethane. It also serves as initial evidence for the notion 
TABLE 2: Composition and physical properties of PUT and PUT/NT-PUT-8 composite films.

\begin{tabular}{lccccc}
\hline Specimens & $\begin{array}{c}\text { Composition } \\
(\text { wt\% } \%\end{array}$ & $\begin{array}{c}T_{g}\left({ }^{\circ} \mathrm{C}\right) \\
(\text { soft segment })\end{array}$ & $\begin{array}{c}\text { Storage modulus } \\
\text { at }-400^{\circ} \mathrm{C}(\mathrm{GPa})\end{array}$ & $\begin{array}{c}\text { Tensile strength } \\
(\mathrm{KPa})\end{array}$ & $\begin{array}{c}\text { Elongation at } \\
\text { break }(\%)\end{array}$ \\
\hline PUT & $100 / 0$ & -26 & 0.918 & 180 & 195 \\
PUT/NT-PUT-8-1 & $100 / 1$ & -24 & 1.14 & 286 & 192 \\
PUT/NT-PUT-8-5 & $100 / 5$ & -19 & 1.22 & 344 & 173 \\
PUT/NT-PUT-8-10 & $100 / 10$ & -17 & 1.47 & 675 & 116 \\
\hline
\end{tabular}

that functionalized carbon nanotubes can be dispersed homogeneously into polymeric matrices. According to TGA results shown in Figure 5, it is obvious that the thermal stability of nanocomposites increases with increasing amounts of PUT-functionalized carbon nanotubes. As a result, the degradation temperature $\left(\mathrm{ca} .335^{\circ} \mathrm{C}\right)$ of $10 \%$ weight loss for PUT/NT-PUT-8-10 composite is ca. $16^{\circ} \mathrm{C}$ higher than that of neat PUT. In addition, compared to the char yield (ca. $3.5 \%$ ) of neat PUT, the higher char yield (ca. 16.9\%) for this composite demonstrates that the effect of enhanced thermal stability due to the PUT-functionalized MWNTs may occur.

3.6. Mechanical Properties of Polyurethane Nanocomposites. Dynamic mechanical analysis (DMA) is most useful for studying the viscoelastic behavior of polymers. The mechanical properties and stiffness of the polyurethane nanocomposites were then analyzed with DMA. In the polyurethane composite, PUT-functionalized MWNTs serve as the reinforcement and segmented PUT acts as the matrix. Increasing the amount of NT-PUT- 8 in the composite increased the storage modulus due to stronger intermolecular interactions between MWNT-PUT nanohybrid and PUT. The soft segment $T_{g}$ s of the three composite films were also obtained from loss tangent of DMA. As shown in Figure 6, the dynamic storage modulus $\left(\mathrm{E}^{\prime}\right)$ and loss tangent $(\tan \delta)$ of the PUT/NT-PUT- 8 nanocomposite films show enhanced mechanical properties and increased soft segment $T_{g}$. The results of soft segment $T_{g}$ of the three composites are listed in Table 2. The enhanced E's of PUT/NT-PUT-8 nanocomposites are induced from the stiffening effect of the CNTs. $E^{\prime}$ of the PUT/NT-PUT-8 nanocomposite films prepared in this study increases with increasing NT-PUT-8 content, which is due to the stiffening effect of the NT-PUT8. In comparison with the $\mathrm{E}^{\prime}$ values of PUT, the $\mathrm{E}^{\prime} \mathrm{s}$ of the three nanocomposites are significantly improved, indicating a strong adhesion between the reinforcement and the matrix. For the convenience of comparison, data of $\mathrm{E}^{\prime}$ at $-40^{\circ} \mathrm{C}$ are also shown in Table 2. Moreover, with increasing NT-PUT-8 content, the glass-transition temperature of the soft segments of the PUD/NT-PUT-8 nanocomposite films shifts from -26 to $-17^{\circ} \mathrm{C}$. The increase of soft segment $T_{g}$ is attributed to the constraint of polyurethane chains by carbon nanotubes. This means that NT-PUT-8 nanohybrids are compatible with the amorphous regions of the soft segments in PUT matrix.

Figure 7 shows the stress-strain curves of the PUT film and PUT/NT-PUT-8 nanocomposite films. The results of tensile strength and elongation at break of the PUD/NT-

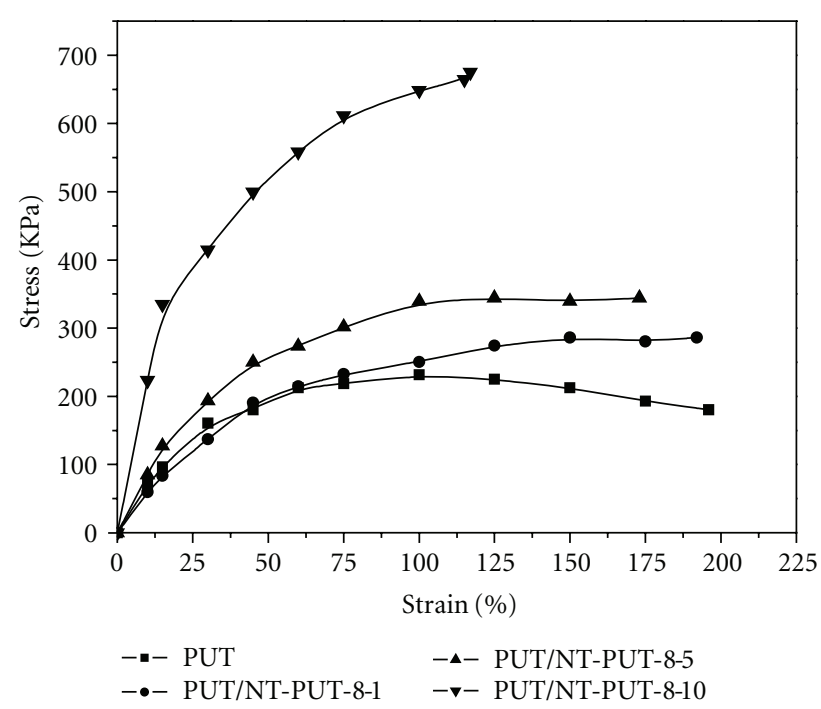

FIgURE 7: Stress-strain curves for PUT and PUT/NT-PUT-8 composite films.

PUT-8 nanocomposite films are also summarized in Table 2. The tensile strengths of the nanocomposite films are enhanced with $1 \mathrm{wt} \%$ to $10 \mathrm{wt} \%$ loading of NT-PUT- 8 compared to the corresponding value of the original PUT film. As the NT-PUT- 8 content increased from $1 \mathrm{wt} \%$ to $10 \mathrm{wt} \%$, the tensile strength of the PUT/NT-PUT- 8 nanocomposite films increases from 286 to $675 \mathrm{KPa}$, corresponding to an increasing ratio of 59 to $275 \%$; however, the elongation at break (\% of strain) decreased from 192 to $116 \%$. The increase of tensile strength in the PUT/NT-PUT-8 nanocomposites is due to the reinforcing effect of NT-PUT-8 in the PUT matrix.

\section{Conclusions}

MWNTs were covalently functionalized with segmented polyurethanes using the "grafting to" technique. The segmented polyurethane (PUT) with hydroxyl groups pendant on the polymer backbone was synthesized by the conventional prepolymer technique. The functionalized MWNT-COCl was then reacted with polyurethane to prepare the MWNTpolyurethane nanohybrids. By XPS analysis, the presence of $\mathrm{C}(=\mathrm{O})(1 \mathrm{~s})$ for acid-treated MWNTs indicated the successful oxidization of MWNTs. From the characteristic peaks of PUT shown in IR spectra, PUT has been grafted to the surfaces of MWNTs successfully. TGA results indicated that 
acid treatment time for $8 \mathrm{~h}$ might be enough. SEM investigations gave direct evidence of the nanostructures of the MWNT-PUT hybrids. The MWNT-PUT nanohybrids were well dispersed in the same solvents for neat PU, thus allowing the intimate mixing of the functionalized nanotubes with the matrix polymer for the preparation of nanocomposites. Dynamic mechanical analysis showed the storage modulus and the soft segment $T_{g}$ of the nanocomposites increased with increasing NT-PUT- 8 content. The tensile strengths of the nanocomposite films with different weight ratio loading of NT-PUT- 8 were enhanced by about 59 to $275 \%$, compared to the corresponding value of the original PUT film.

\section{References}

[1] A. L. Martínez-Hernández, C. Velasco-Santos, and V. M. Castaño, "Carbon nanotubes composites: processing, grafting and mechanical and thermal properties," Current Nanoscience, vol. 6, no. 1, pp. 12-39, 2010.

[2] T. Kashiwagi, E. Grulke, J. Hilding et al., "Thermal and flammability properties of polypropylene/carbon nanotube nanocomposites," Polymer, vol. 45, no. 12, pp. 4227-4239, 2004.

[3] W. Bauhofer and J. Z. Kovacs, "A review and analysis of electrical percolation in carbon nanotube polymer composites," Composites Science and Technology, vol. 69, no. 10, pp. 14861498, 2009.

[4] B. Fan, X. Mei, K. Sun, and J. Ouyang, "Conducting polymer/carbon nanotube composite as counter electrode of dyesensitized solar cells," Applied Physics Letters, vol. 93, no. 14, Article ID 143103, 2008.

[5] M. S. Dresselhaus, G. Dresselhaus, and P. C. Eklund, Science of Fullerenes and Carbon Nanotubes, Academic Press, New York, NY, USA, 1996.

[6] R. Saito, M. S. Dresselhaus, and G. Dresselhaus, Physical Properties of Carbon Nanotubes, Imperial College Press, London, UK, 1998.

[7] P. M. Ajayan, L. S. Schadler, C. Giannaris, and A. Rubio, "Single-walled carbon nanotube-polymer composites: strength and weakness," Advanced Materials, vol. 12, no. 10, pp. 750-753, 2000.

[8] B. Ni and S. B. Sinnott, "Chemical functionalization of carbon nanotubes through energetic radical collisions," Physical Review B, vol. 61, no. 24, pp. R16343-R16346, 2000.

[9] J. Chen, M. A. Hamon, H. Hu et al., "Solution properties of single-walled carbon nanotubes," Science, vol. 282, no. 5386, pp. 95-98, 1998.

[10] R. H. Baughman, A. A. Zakhidov, and W. A. De Heer, "Carbon nanotubes-the route toward applications," Science, vol. 297, no. 5582, pp. 787-792, 2002.

[11] X. Chen and S. P. Armes, "Surface polymerization of hydrophilic methacrylates from ultrafine silica sols in protic media at ambient temperature: a novel approach to surface functionalization using a polyelectrolytic macroinitiator," Advanced Materials, vol. 15, no. 18, pp. 1558-1562, 2003.

[12] A. Hirsch, "Functionalization of single-walled carbon nanotubes," Angewandte Chemie International Edition, vol. 41, no. 11, pp. 1853-1859, 2002.

[13] Y. P. Sun, K. Fu, Y. Lin, and W. Huang, "Functionalized carbon nanotubes: properties and applications," Accounts of Chemical Research, vol. 35, no. 12, pp. 1096-1104, 2002.

[14] R. Czerw, Z. Guo, P. M. Ajayan, Y. P. Sun, and D. L. Carroll, "Organization of polymers onto carbon nanotubes: a route to nanoscale assembly," Nano Letters, vol. 1, no. 8, pp. 423-427, 2001.

[15] S. Qin, D. Qin, W. T. Ford, D. E. Resasco, and J. E. Herrera, "Functionalization of single-walled carbon nanotubes with polystyrene via grafting to and grafting from methods," Macromolecules, vol. 37, no. 3, pp. 752-757, 2004.

[16] Y. Lin, B. Zhou, K. A. S. Fernando, P. Liu, L. F. Allard, and Y. P. Sun, "Polymeric carbon nanocomposites from carbon nanotubes functionalized with matrix polymer," Macromolecules, vol. 36, no. 19, pp. 7199-7204, 2003.

[17] M. S. P. Shaffer and K. Koziol, "Polystyrene grafted multiwalled carbon nanotubes," Chemical Communications, no. 18, pp. 2074-2075, 2002.

[18] W. Wu, S. Zhang, Y. Li et al., "PVK-modified single-walled carbon nanotubes with effective photoinduced electron transfer," Macromolecules, vol. 36, no. 17, pp. 6286-6288, 2003.

[19] H. Kong, C. Gao, and D. Yan, "Controlled functionalization of multiwalled carbon nanotubes by in situ atom transfer radical polymerization," Journal of the American Chemical Society, vol. 126, no. 2, pp. 412-413, 2004.

[20] I. C. Liu, H. M. Huang, C. Y. Chang, H. C. Tsai, C. H. Hsu, and R. C. C. Tsiang, "Preparing a styrenic polymer composite containing well-dispersed carbon nanotubes: anionic polymerization of a nanotube-bound p-methylstyrene," Macromolecules, vol. 37, no. 2, pp. 283-287, 2004.

[21] S. L. Ruan, P. Gao, X. G. Yang, and T. X. Yu, “Toughening high performance ultrahigh molecular weight polyethylene using multiwalled carbon nanotubes," Polymer, vol. 44, no. 19, pp. 5643-5654, 2003.

[22] W. Tang, M. H. Santare, and S. G. Advani, "Melt processing and mechanical property characterization of multi-walled carbon nanotube/high density polyethylene (MWNT/HDPE) composite films," Carbon, vol. 41, no. 14, pp. 2779-2785, 2003.

[23] S. A. Gordeyev, F. J. MacEdo, J. A. Ferreira, F. W. J. Van Hattum, and C. A. Bernardo, "Transport properties of polymer-vapour grown carbon fibre composites," Physica B, vol. 279, no. 1-3, pp. 33-36, 2000.

[24] Z. Jia, Z. Wang, C. Xu et al., "Study on poly(methyl methacrylate)/carbon nanotube composites," Materials Science and Engineering A, vol. 271, no. 1-2, pp. 395-400, 1999.

[25] Z. Jin, K. P. Pramoda, G. Xu, and S. H. Goh, "Dynamic mechanical behavior of melt-processed multi-walled carbon nanotube/poly(methyl methacrylate) composites," Chemical Physics Letters, vol. 337, no. 1-3, pp. 43-47, 2001.

[26] D. Qian, E. C. Dickey, R. Andrews, and T. Rantell, "Load transfer and deformation mechanisms in carbon nanotubepolystyrene composites," Applied Physics Letters, vol. 76, no. 20, pp. 2868-2870, 2000.

[27] R. Andrews, D. Jacques, A. M. Rao et al., "Nanotube composite carbon fibers," Applied Physics Letters, vol. 75, no. 9, pp. 13291331, 1999.

[28] L. S. Schadler, S. C. Giannaris, and P. M. Ajayan, "Load transfer in carbon nanotube epoxy composites," Applied Physics Letters, vol. 73, no. 26, pp. 3842-3844, 1998.

[29] J. M. Park, D. S. Kim, J. R. Lee, and T. W. Kim, "Nondestructive damage sensitivity and reinforcing effect of carbon nanotube/epoxy composites using electro-micromechanical technique," Materials Science and Engineering C, vol. 23, no. 6-8, pp. 971-975, 2003.

[30] C. Gao, Y. Z. Jin, H. Kong et al., "Polyurea-functionalized multiwalled carbon nanotubes: synthesis, morphology, and Raman spectroscopy," Journal of Physical Chemistry B, vol. 109, no. 24, pp. 11925-11932, 2005. 
[31] H. Xia and M. Song, "Preparation and characterisation of polyurethane grafted single-walled carbon nanotubes and derived polyurethane nanocomposites," Journal of Materials Chemistry, vol. 16, no. 19, pp. 1843-1851, 2006.

[32] J. Kwon and H. Kim, "Comparison of the properties of waterborne polyurethane/multiwalled carbon nanotube and acidtreated multiwalled carbon nanotube composites prepared by in situ polymerization," Journal of Polymer Science Part A, vol. 43, no. 17, pp. 3973-3985, 2005.

[33] J. Kwon and H. Kim, "Preparation and properties of acidtreated multiwalled carbon nanotube/waterborne polyurethane nanocomposites," Journal of Applied Polymer Science, vol. 96, no. 2, pp. 595-604, 2005.

[34] T. L. Wang and C. G. Tseng, "Polymeric carbon nanocomposites from multiwalled carbon nanotubes functionalized with segmented polyurethane," Journal of Applied Polymer Science, vol. 105, no. 3, pp. 1642-1650, 2007.

[35] D. A. Skoog and F. J. Holler, Principles of Instrumental Analysis, Saunders, Philadelphia, Pa, USA, 5th edition, 1992.

[36] E. Y. Kim, J. S. Kong, S. K. An, and H. D. Kim, "Surface modification of polymers and improvement of the adhesion between evaporated copper metal film and a polymer. I. Chemical modification of PET," Journal of Adhesion Science and Technology, vol. 14, no. 9, pp. 1119-1130, 2000. 

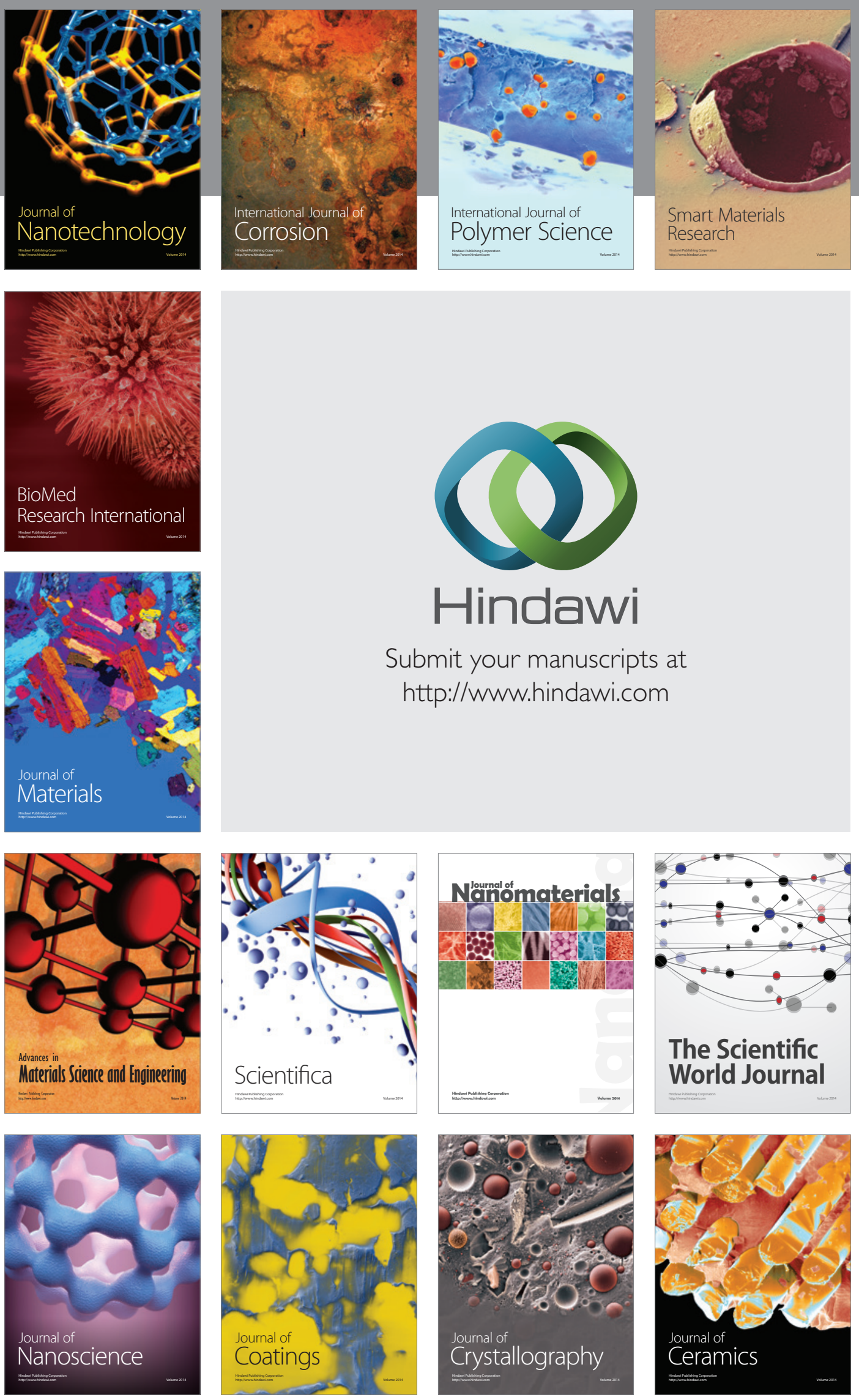

The Scientific World Journal

Submit your manuscripts at

http://www.hindawi.com

\section{World Journal}

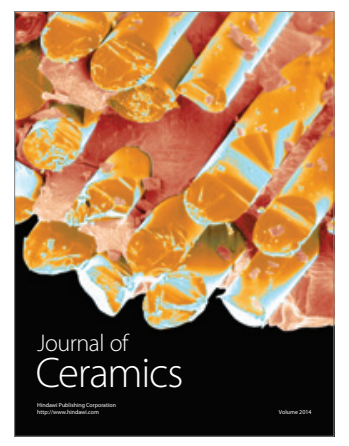

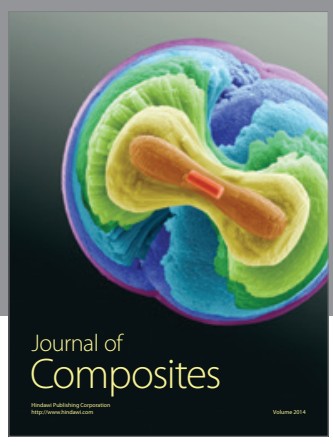
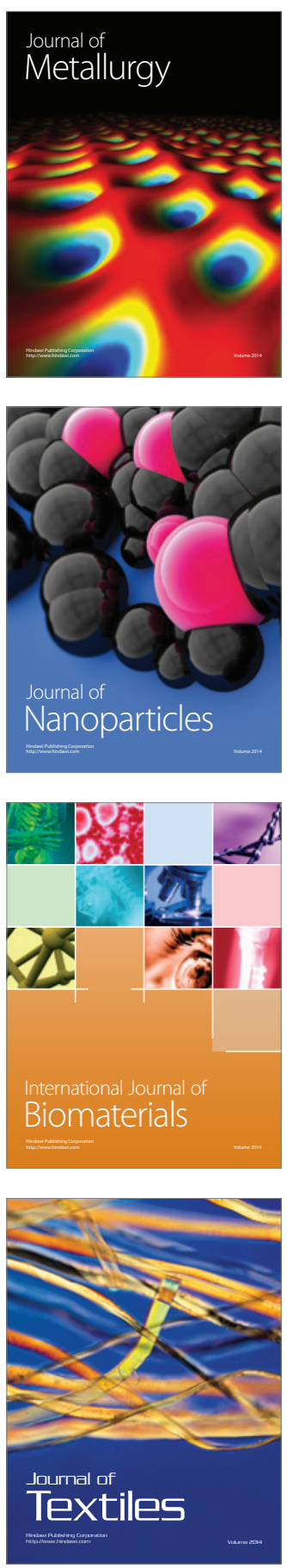